Research Paper

\title{
High delta-like ligand 4 expression correlates with a poor clinical outcome in gastric cancer
}

\author{
Youjin Kim ${ }^{1,2^{*}}$, Sun-ju Byeon ${ }^{3,4^{*}}$, Joonyoung Hur ${ }^{1}$, Kangkook Lee ${ }^{1}$, Dongin Kim ${ }^{5}$, Jin-Hyung Ahn ${ }^{5}$, Sang \\ Hoon Lee ${ }^{5}$, Weon-Kyoo You ${ }^{5}$, Seung Tae Kim ${ }^{1}$, Se Hoon Park ${ }^{1}$, Won Ki Kang ${ }^{1}$, Kyoung-Mee Kim ${ }^{\circledR}$, Jeeyun \\ Lee $^{1 \bowtie}$ \\ 1. Division of Hematology-Oncology, Department of Medicine, Samsung Medical Center, Sungkyunkwan University School of Medicine, Seoul, Korea \\ 2. Division of Hematology-Oncology, Department of Medicine, Samsung Changwon Hospital, Sungkyunkwan University School of Medicine, Changwon, \\ Korea \\ 3. Department of Pathology and Translational Genomics, Samsung Medical Center, Sungkyunkwan University School of Medicine, Seoul, Korea \\ 4. Department of Pathology, Hallym University Dongtan Sacred Heart Hospital, Korea \\ 5. ABL Bio, Korea \\ *Equal contributing authors
}

$\triangle$ Corresponding authors: Jeeyun Lee, MD, Division of Hematology-Oncology, Department of Medicine, Samsung Medical Center, Sungkyunkwan University School of Medicine, 81 Irwon-ro, Gangnam-gu, Seoul, 06351 (Korea). Tel. +82 23410 1779; Fax +82 23410 1754; Email: jyunlee@skku.edu and Kyoung-Mee Kim, MD, Department of Pathology and Translational Genomics, Samsung Medical Center, Sungkyunkwan University School of Medicine, 81 Irwon-ro, Gangnam-gu, Seoul, 06351 (Korea). Tel. +82 23410 2807; Fax +82 23410 1754; Email: kkmkys@skku.edu

(C) Ivyspring International Publisher. This is an open access article distributed under the terms of the Creative Commons Attribution (CC BY-NC) license (https://creativecommons.org/licenses/by-nc/4.0/). See http://ivyspring.com/terms for full terms and conditions.

Received: 2018.09.28; Accepted: 2019.04.30; Published: 2019.06.02

\begin{abstract}
Background: Emerging evidence suggests that delta-like ligand 4 (DLL4) and other members of the Notch pathway may offer new targets for development of anti-angiogenesis drugs for the treatment of several tumor types. However, the role of DLL4 in gastric cancer (GC) remains unclear. In this study, we investigated the impact of DLL4 overexpression on recurrence and survival in gastric cancer (GC) patients.

Methods: DLL4 expression levels were evaluated by immunohistochemistry in tissue samples from 336 GC patients. Samples were classified into high and low DLL4 expression according to a cut-off of $50 \%$ positively stained cells. The correlation between DLL4 expression and clinicopathological parameters, disease-free survival (DFS), and overall survival (OS) were statistically analyzed.

Results: High DLL4 expression was observed in 67 (19.9\%) of the 336 GC patients. After a median follow-up duration of 54.97 months [ $95 \%$ confidence interval (Cl), 52.40-57.55 months), patients at stage II-IV with high DLL4 expression showed significantly poorer DFS compared with those at the same stage but with low DLL4 expression [not reached (NR) for both cohorts, hazard ratio (HR) $0.73(95 \% \mathrm{Cl}$, $0.38-1.40$ ); $p=0.007]$. Likewise, GC patients with high DLL4 expression had a significantly shorter OS following curative surgery compared to those with low DLL4 expression [NR for both groups, HR 0.56 $(95 \% \mathrm{Cl}, 0.32-0.96 ; p=0.002]$. High DLL4 expression had a greater influence on DFS in stage Illb/lV patients than in patients at early stages [34.87 vs. 10.1 months; $\mathrm{HR}, 0.44(95 \% \mathrm{Cl}, 0.19-0.96) ; p=0.004$ ]. Moreover, stage IIIb/IV patients with high DLL4 expression had a significantly shorter OS after surgery than those with low DLL4 expression [58.87 vs. 16.93 months, HR 0.39 ( $95 \% \mathrm{Cl}, 0.16-0.99), p=0.001)$.

Conclusion: High DLL4 expression was observed in $19.9 \%$ of GC patients and was significantly associated with poor survival following curative surgery. Given its prevalence in the GC cohort with a poor prognosis, DLL4 is a potential therapeutic target.
\end{abstract}

Key words: DLL4, Gastric cancer, anti-angiogenesis

\section{Introduction}

In 2012, approximately 951,000 individuals were diagnosed with gastric cancer (GC), which led to the death of 723,000 individuals worldwide [1]. Most patients are diagnosed with GC at a late stage when 
symptoms appear, and thus the prognosis is poor [2]: the median survival time for recurrent or metastatic GC remains less than 1 year. Recently, anti-angiogenesis agents have emerged as efficacious anti-tumor drugs against several tumor types, including colorectal cancer and GC. The RAINBOW trial, which compared the effects of paclitaxel with or without the anti-vascular endothelial factor receptor 2 (VEGFR2) monoclonal antibody ramucirumab in second-line chemotherapy for metastatic GC resulted in improved survival in the paclitaxel/ramucirumab arm [3]. Accordingly, the paclitaxel/ramucirumab combination is the current standard chemotherapy regimen for GC in a second-line setting. Because the prognosis remains poor after recurrence following curative surgery, identification of prognostic biomarkers associated with pivotal signaling pathways is crucial for enhancing survival for GC patients $\{$ Kim, 2018 \#2680\}. The most widely prescribed anti-angiogenesis inhibitor is bevacizumab, which is a monoclonal antibody against the human VEGF ligand approved for use in the treatment colorectal and ovarian cancers. VEGF/VEGFR signaling inhibitors block VEGF-driven angiogenesis and thus regress VEGF-dependent tumor vessels. Nevertheless, VEGF inhibitors alone do not destroy all of the blood vessels in tumors. Indeed, clinical studies have demonstrated the emergence of resistance to anti-VEGF monotherapy $[4,5]$.

A few studies have identified the Notch signaling pathway as a newly emerging therapeutic tumor angiogenesis target [6-8]. Five ligands in the Notch signaling pathway (Jagged 1 and 2 and delta-like ligands 1, 3, and 4) are involved in tumorigenesis, tumor progression, tumor angiogenesis, and chemo-resistance. Among these, delta-like ligand 4 (DLL4) has received the most attention, and DLL4 inhibitors have been developed that show potent anti-tumor effects in a broad spectrum of VEGF therapy-resistant xenograft models [9-12]. Nevertheless, the prevalence of DLL4 expression in tumor specimens and its clinical implication in GC has not been extensively studied. Since this information is essential for identifying the potential target population for anti-DLL4 inhibitor treatment, in the present study, we surveyed the expression level of DLL4 using immunohistochemical (IHC) analysis in samples obtained from a large and well-defined cohort from the Adjuvant Chemoradiotherapy in Stomach Tumors (ARTIST) trial [13], and evaluated the association of DLL4 expression with patient survival.

\section{Materials and Methods}

\section{Patients}

We used the ARTIST trial results for our analysis. The complete details of the ARTIST trial are published elsewhere [13]. In brief, 458 patients with pathological stage Ib-IV (M0) GC, according to the American Joint Committee on Cancer 2002 staging system, were randomly assigned to receive the adjuvant XP $(n=228)$ or XPRT $(n=230)$ treatment. The treatment regimen administered in the XP arm included six cycles of $1,000 \mathrm{mg} / \mathrm{m}^{2}$ capecitabine administered twice daily on days $1-14$ and $60 \mathrm{mg} / \mathrm{m}^{2}$ cisplatin on day 1 . The treatment regimen administered in the XPRT arm included concomitant administration of a total radiotherapy dose of $45 \mathrm{~Gy}$ and $825 \mathrm{mg} / \mathrm{m}^{2}$ capecitabine twice daily after completion of two cycles of $\mathrm{XP}$, followed by administration of two additional cycles of XP. Written informed consent was obtained from all participating patients before they were randomly assigned to groups. A total of 336 specimens were available for further analysis in the present study.

\section{IHC analysis}

Representative tumor areas were selected for all available samples, and a tissue microarray was constructed after reviewing a hematoxylin and eosin-stained section from the donor block. Using this slide for guidance, two representative regions of the tumor were sampled from the donor block, and 2-mm-diameter cores were extracted and embedded in the array block. Tumor sections from the array blocks were freshly cut into $3-\mu \mathrm{m}$ slices, and dried at $60^{\circ} \mathrm{C}$ for $30 \mathrm{~min}$. For DLL4 IHC, the slides were deparaffinized, and endogenous peroxidase was blocked for $5 \mathrm{~min}$. Antigen retrieval was performed in an autoimmunostainer at $97^{\circ} \mathrm{C}$ for $20 \mathrm{~min}$ with ER1 buffer ( $\mathrm{pH}$ 6.0). The slides were incubated with mouse DLL4 monoclonal antibody (HPA023392, 1:150, Sigma-Aldrich) at room temperature for $15 \mathrm{~min}$ using a BOND-MAX autoimmunostainer (Leica Microsystems, Wetzlar, Germany) and BOND Polymer Refine Detection kit (DS9800; Vision BioSystems, Melbourne, Australia).

Two well-experienced pathologists examined the IHC slides without prior information about the clinicopathological features of the patients. Semiquantitative estimates were achieved using a composite score comprised of the sum of the staining intensity values and the relative abundance of positive cells. The intensities were graded as 0 (negative), 1 (weakly positive), 2 (moderately positive), and 3 (strongly positive). The abundance of positive cells was graded from 0 to $4(0<5 \%$ positive 
cells; 1, 5-24 \% 2, 25-49 \% ; 3, 50-74 \% ; 4, 75-100 \%). Based on the distribution of results, low and high DLL4 expression was defined if $<50 \%$ or $\geq 50 \%$ of the cells were stained with DLL4 monoclonal antibody, respectively (Fig. 1).

\section{Statistical analyses}

The correlations between DLL4 expression and clinical characteristics were analyzed using the $\chi^{2}$ test or t-test as appropriate. In univariate survival analysis, the probability of disease-free survival (DFS) or overall survival (OS) was determined by the Kaplan-Meier method. Because the median survival was not reached in several subgroups, Kaplan-Meier estimates of the 5-year DFS and 5-year OS are presented. Different survival curves were compared using the log-rank test. Multivariate survival analysis was performed using the Cox proportional hazards regression model, including all variables that were significantly associated with patient survival in the univariate analysis along with patient age as an additional parameter. In general, $p$ values $<0.05$ were considered statistically significant. For statistical evaluation, we used the SPSS software package (version 10.0; SPSS, Inc., Chicago, IL, USA).

\section{Results}

\section{Relation of DLL4 expression with patient characteristics}

Of the 458 patients enrolled in the ARTIST trial, 336 had stage II, III, or IV GC, and these samples were included for analysis in the present study. The demographic and baseline characteristics of these patients are summarized in Table 1 . The median post-operative follow-up period of this cohort was 54.97 [95\% confidence interval (CI) 52.40-57.55] months, with an observed mortality rate of $26.6 \%$ $(89 / 336)$ after radical gastrectomy. The majority of patients were over 50 years of age and were men.

High DLL4 expression was detected in 67 $(19.9 \%)$ of the 336 GC patients. Figure 1 provides representative IHC staining images of DLL4 in GC tissues. The $\chi^{2}$ analysis showed that patients with Lauren's intestinal-type GC had higher expression of DLL4 than those with diffuse-type GC $(p<0.001)$. In addition, a significant correlation was observed between DLL4 expression and HER2 status $(p=$ $0.018)$. However, there was no significant correlation between DLL4 expression and other clinicopathological characteristics such as age, sex, tumor localization, Borrmann's classification, tumor status $(\mathrm{pT})$, lymph node metastasis $(\mathrm{pN})$, perineural invasion, or lymphovascular invasion (all $p>0.05$ ).
Table 1. Patients and disease characteristics in the gastric cancer and according to DLL4 status.

\begin{tabular}{|c|c|c|c|c|}
\hline Characteristic & $\mathrm{N}=336(\%)$ & $\begin{array}{l}\text { DLL } 4 \text { (low) } \\
\text { n=269 (80.1\%) }\end{array}$ & $\begin{array}{l}\text { DLL } 4 \text { (high) } \\
\mathrm{n}=67(19.9 \%)\end{array}$ & $p$ value \\
\hline \multicolumn{5}{|l|}{ Age } \\
\hline Median (range), years & $57(28-77)$ & $56(28-77)$ & $59(33-77)$ & 0.104 \\
\hline$<60$ & $195(58.0)$ & $162(60.2)$ & $33(49.3)$ & \\
\hline$>60$ & $141(42.0)$ & $107(39.8)$ & $34(50.7)$ & \\
\hline \multicolumn{5}{|l|}{ Sex } \\
\hline Male & 217 (64.6) & $172(63.9)$ & $45(67.2)$ & 0.622 \\
\hline Female & $119(35.4)$ & $97(36.1)$ & $22(32.8)$ & \\
\hline Tumor Localization & & & & 0.296 \\
\hline Cardias & $17(5.1)$ & $12(4.5)$ & $5(7.5)$ & \\
\hline Body & $159(47.3)$ & $133(49.4)$ & $26(38.7)$ & \\
\hline Antrum & $125(37.2)$ & $95(35.3)$ & $30(44.8)$ & \\
\hline Whole/multiple & $35(10.4)$ & $29(10.8)$ & $6(9.0)$ & \\
\hline Histology & & & & 0.042 \\
\hline $\mathrm{ADC}, \mathrm{WD}$ & $5(1.5)$ & $2(0.7)$ & $3(4.5)$ & \\
\hline $\mathrm{ADC}, \mathrm{MD}$ & $109(32.4)$ & $83(30.9)$ & $26(38.8)$ & \\
\hline $\mathrm{ADC}, \mathrm{PD}$ & $147(43.8)$ & $119(44.2)$ & $28(41.8)$ & \\
\hline Signet ring cell & $75(22.3)$ & $65(24.2)$ & $10(14.9)$ & \\
\hline \multicolumn{5}{|c|}{ Borrman classification } \\
\hline 1 & $7(2.1)$ & $4(1.5)$ & $3(4.5)$ & 0.308 \\
\hline 2 & $98(29.2)$ & $77(28.6)$ & $21(31.3)$ & \\
\hline 3 & $193(57.4)$ & $156(58.0)$ & $37(55.2)$ & \\
\hline 4 & $38(11.3)$ & $32(11.9)$ & $6(9.0)$ & \\
\hline \multicolumn{5}{|l|}{ Lauren type } \\
\hline Intestinal & $132(39.2)$ & $92(34.2)$ & $40(59.7)$ & $<0.001$ \\
\hline Diffuse & $189(56.3)$ & $164(61.0)$ & $25(37.3)$ & \\
\hline Mixed & $15(4.5)$ & $13(4.8)$ & $2(3.0)$ & \\
\hline \multicolumn{5}{|l|}{ pT stage } \\
\hline 1 & $8(2.4)$ & $7(2.6)$ & $1(1.5)$ & 0.511 \\
\hline $2 a$ & $64(19.0)$ & $54(20.1)$ & $10(14.9)$ & \\
\hline $2 b$ & $158(47.0)$ & $120(44.6)$ & $38(56.7)$ & \\
\hline 3 & $95(28.3)$ & $79(29.4)$ & $16(23.9)$ & \\
\hline 4 & $11(3.3)$ & $9(3.3)$ & $2(3.0)$ & \\
\hline \multicolumn{5}{|l|}{ pN stage } \\
\hline 0 & $13(3.9)$ & $11(4.1)$ & $2(3.0)$ & 0.130 \\
\hline 1 & $202(60.1)$ & $165(61.3)$ & 37 (55.2) & \\
\hline 2 & $87(25.9)$ & $71(26.4)$ & $16(23.9)$ & \\
\hline 3 & $34(10.1)$ & $22(8.2)$ & $12(17.9)$ & \\
\hline Stage & & & & 0.037 \\
\hline II and IIIa & $271(80.7)$ & $223(82.9)$ & 48 (71.6) & \\
\hline IIIb and IV & 65 (19.3) & $46(17.1)$ & $19(28.4)$ & \\
\hline Cer-b status & & & & 0.018 \\
\hline negative & $281(83.6)$ & $226(84.0)$ & $55(82.1)$ & \\
\hline positive & $18(5.4)$ & $9(3.3)$ & $9(13.4)$ & \\
\hline unknown & $37(11.0)$ & $34(12.6)$ & $3(4.5)$ & \\
\hline Surgical resection & & & & 0.929 \\
\hline Subtotal & $199(59.2)$ & $159(59.1)$ & $40(59.7)$ & \\
\hline Total & $137(40.8)$ & $110(40.9)$ & $27(40.3)$ & \\
\hline \multicolumn{5}{|l|}{ Perineural invasion } \\
\hline No & $177(52.5)$ & $143(50.3)$ & $34(50.7)$ & 0.743 \\
\hline Yes & 159 (47.5) & $126(47)$ & $33(49.3)$ & \\
\hline \multicolumn{5}{|l|}{$\begin{array}{l}\text { Lymphovascular } \\
\text { invasion }\end{array}$} \\
\hline No & $106(31.5)$ & $86(32.0)$ & $20(29.9)$ & 0.738 \\
\hline Yes & $230(68.5)$ & $183(68.0)$ & $47(70.1)$ & \\
\hline
\end{tabular}

\section{Impact of DLL4 expression on patient survival}

Kaplan-Meier survival curves were plotted separately for all patients $(\mathrm{n}=336$; (Fig. 2A, B), for those with stages II and IIIa GC $(n=269$; Fig. 2 C, D), and for those with stages IIIb and IV GC ( $n=67$; Fig. 2E, F). Stage II-IV GC patients with high DLL4 expression had significantly poorer DFS compared with those with low DLL4 expression [median DFS, not reached (NR) for both cohorts, hazard ratio (HR) 0.73 (95\% CI, 0.38-1.40); $p=0.007]$, and the 5-year DFS 
rate of GC patients with low DLL4 expression (75.9\%) was significantly better than that of patients harboring tumors with high DLL4 expression (63.5\%). In addition, statistically significant differences in OS were observed for stage II-IV GC patients according to DLL4 expression, with a poor outcome for patients with a greater proportion of DLL4-stained cells [HR 0.56 (95\% CI, 0.32-0.96), $\boldsymbol{p}=0.002]$. Neither DFS nor OS differed significantly according to DLL4 expression for patients with stage II and IIIa GC [DFS: $\mathrm{HR}=0.73$ (95\% CI, 0.38-1.40), $\boldsymbol{p}=0.176$; median OS: $\mathrm{HR}=0.60$ (95\% CI, 0.30-1.22), $\boldsymbol{p}=0.052]$. However, among patients with stages IIIb and IV GC, significantly shorter DFS and OS was associated with high DLL4 expression [DFS: 34.87 vs. $10.10, \mathrm{HR}=0.44$ (95\% CI, $0.19-0.96), p=0.004$; OS: 58.87 vs. $16.93, \mathrm{HR}=$ 0.39 (95\% CI, 0.16-0.99), $p=0.001]$.

\section{Univariate and multivariate Cox regression analyses}

As shown in Table 2, univariate Cox regression analysis suggested that DLL4 expression, histological differentiation, Borrmann's classification, Lauren's classification, depth of invasion ( $\mathrm{pT}), \mathrm{pN}, \mathrm{TNM}$ stage, and surgical resection were significantly associated with both OS and DFS. Multivariate regression with these eight significant variables further supported that high DLL4 expression, histologically poor differentiation and signet ring cell type, and advanced TNM stage (IIIb and IV) were independent prognostic factors of poor DFS. In addition, high DLL4 expression, Lauren's diffuse type, and advanced TNM stage (IIIb and IV) were independent prognostic factors of OS (Table 2).

Table 2. Prognostic factors associated with DFS and OS as determined by univariate and multivariate analysis

\begin{tabular}{|c|c|c|c|c|c|}
\hline \multicolumn{3}{|l|}{ DFS } & \multicolumn{3}{|l|}{ OS } \\
\hline Variables & $\mathrm{HR}(95 \% \mathrm{CI})$ & $p$-value & Variables & $\mathrm{HR}(95 \% \mathrm{CI})$ & $p$-value \\
\hline Univariate analysis & & & Univariate analysis & & \\
\hline DLL 4 negative vs. positive & $1.93(1.27-2.95)$ & 0.002 & DLL 4 negative vs. positive & $1.95(1.24-3.08)$ & 0.004 \\
\hline *Histological differentiation & $1.72(1.10-2.67)$ & 0.016 & ${ }^{*}$ Histological differentiation & $1.83(1.12-2.99)$ & 0.015 \\
\hline Borrman $(1,2$ vs 3,4$)$ & $1.85(1.33-2.56)$ & $<0.001$ & Borrman $(1,2$ vs 3,4$)$ & $2.16(1.52-3.08)$ & $<0.001$ \\
\hline Lauren intestinal vs. diffuse & $1.53(1.10-2.14)$ & 0.012 & Lauren intestinal vs. diffuse & $1.70(1.08-2.68)$ & 0.019 \\
\hline pT 1,2 vs. pT 3,4 & $2.26(1.54-3.30)$ & $<0.001$ & pT 1,2 vs. pT 3,4 & $2.46(1.62-3.73)$ & $<0.001$ \\
\hline pN 0,1 vs. pN 2,3 & $2.73(1.87-4.00)$ & $<0.001$ & pN 0,1 vs. $\mathrm{pN} 2,3$ & $2.57(1.69-3.90)$ & $<0.001$ \\
\hline Stage(II/IIIa versus IIIb/IV) & $3.82(2.58-5.65)$ & $<0.001$ & Stage(II/IIIa versus IIIb/IV) & $3.55(2.31-4.56)$ & $<0.001$ \\
\hline Surgical resection TG vs STG & $1.70(1.16-2.47)$ & 0.007 & Surgical resection TG vs STG & $1.84(1.22-2.80)$ & 0.004 \\
\hline Multivariate analysis & & & Multivariate analysis & & \\
\hline DLL 4 negative vs. positive & $1.80(1.14-2.86)$ & 0.012 & DLL 4 negative vs. positive & $2.07(1.23-3.46)$ & 0.006 \\
\hline *Histological differentiation & $1.78(1.10-2.89)$ & 0.021 & Lauren intestinal vs. diffuse & $2.19(1.30-3.69)$ & 0.003 \\
\hline Stage(II/IIIa versus IIIb/IV) & $3.95(2.56-6.11)$ & $<0.001$ & Stage(II/IIIa versus IIIb/IV) & $3.93(2.45-6.32)$ & $<0.001$ \\
\hline
\end{tabular}

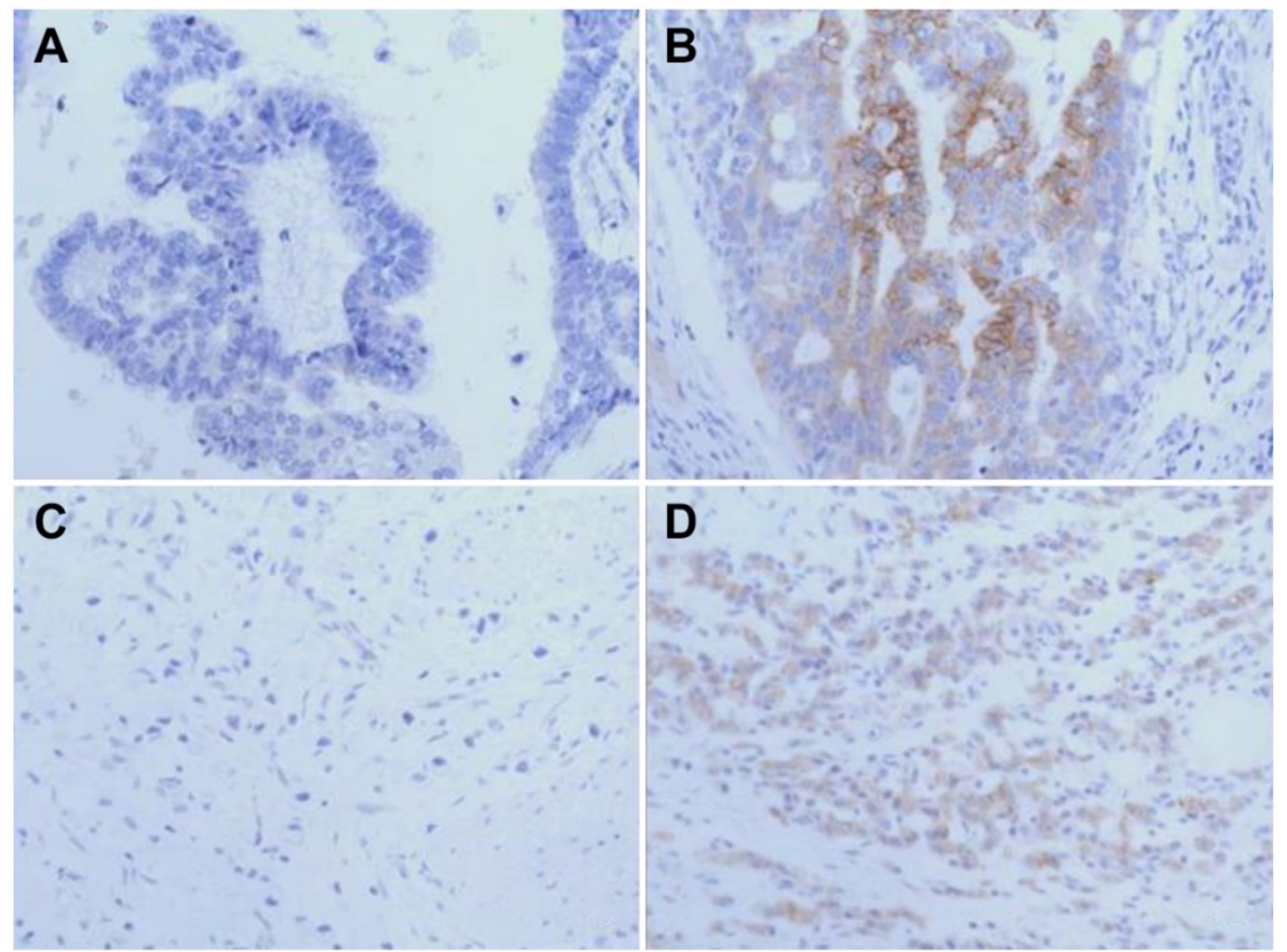

Figure 1. Representative microphotographs of DLL4 immunohistochemistry. (A) low DLL4 expression, intestinal-type; (B) high DLL4 expression, intestinal-type; (C) low DLL4 expression, diffuse-type; and (D) high DLL4 expression, diffuse-type gastric adenocarcinomas 
Stage II - IV

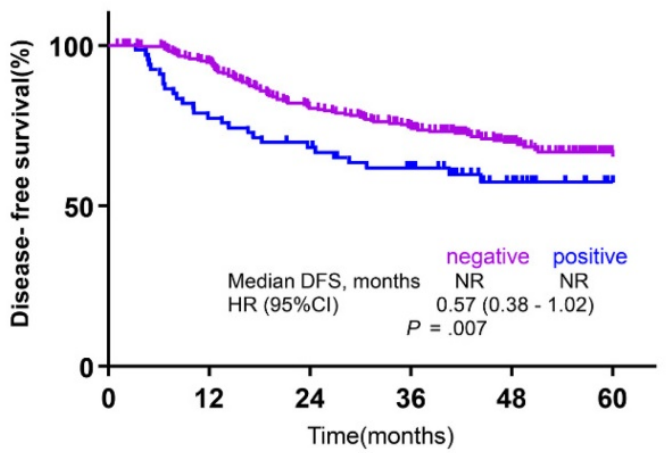

Stage II,IIla

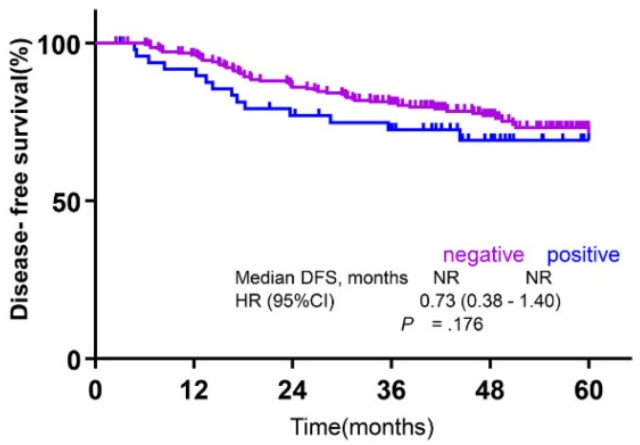

Stage IIIb,IV

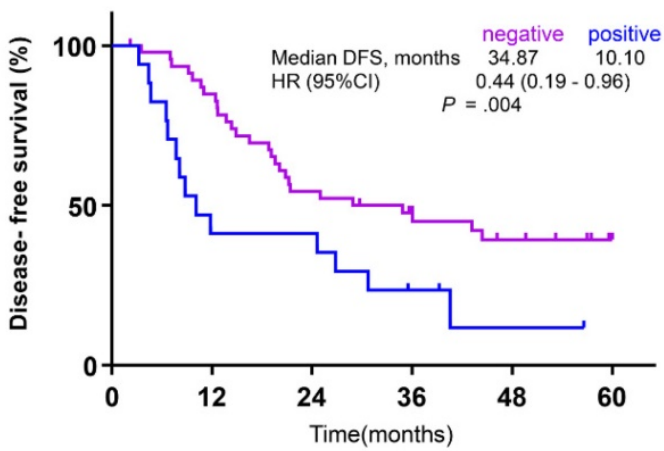

Stage II - IV

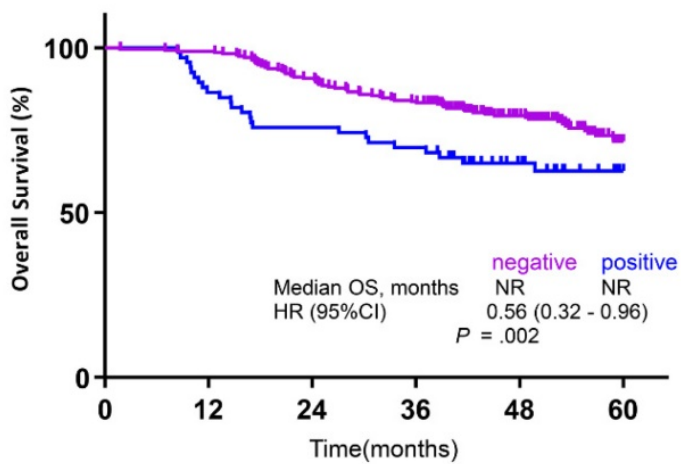

Stage II, IIla

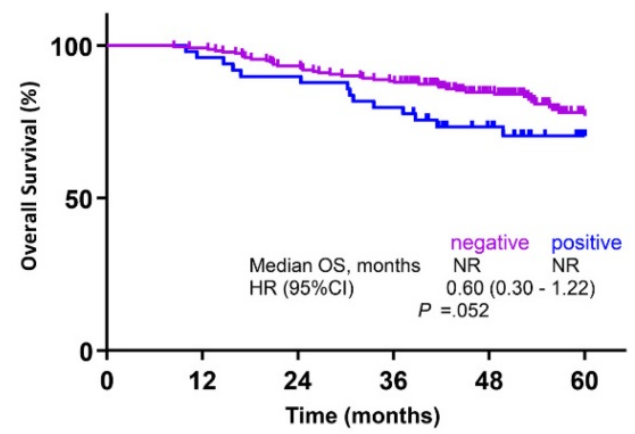

Stage IIIb,IV

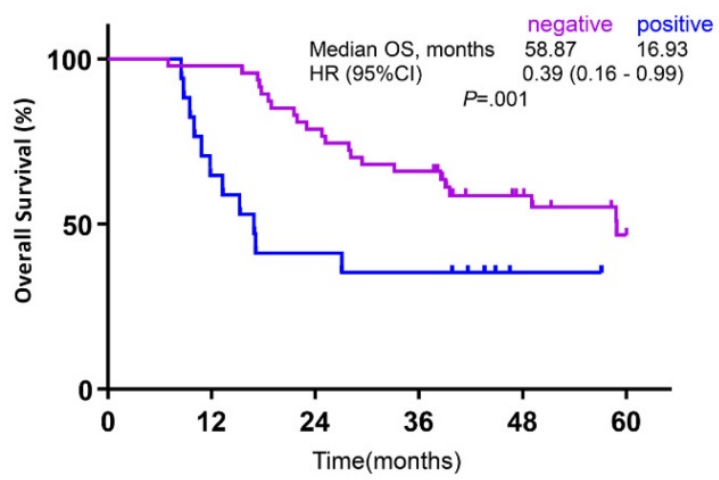

Figure 2. Kaplan-Meier survival analysis according to the clinical stage of gastric cancer (GC). (A) Disease-free survival (DFS) in stage II-IV GC, (B) overall survival (OS) in stage II-IV GC, (C) DFS in stage II-IIla GC, (D) OS in stage II-IIla GC, (E) DFS in stage IIIb-IV GC, and (F) OS in stage IIIB-IV GC

\section{Discussion}

The overall results of this study suggest that high DLL4 expression is associated with a poor DFS and OS of GC patients following surgery. These results further imply that DLL4 expression may be correlated with metastases or recurrence following curative surgery in GC. Indeed, previous studies have shown that overexpression of DLL4 increased the risk of a poor clinical prognosis in other tumor types [14-18]. Thus, DLL4 may be a novel biomarker for predicting the prognosis and recurrence of GC.
Targeting tumor cell-specific DLL4 is a feasible anti-angiogenic treatment strategy by disrupting DLL4 in the tumor vasculature, along with direct effects on the frequency of tumor-initiating cells and tumor growth, either alone or in combination with chemotherapy [10]. Increased Notch1 activity has been reported in intestinal tumors [19]; in addition, we observed a high proportion of GC samples with high DLL4 expression. Taken together, these data implicate a role of DLL4 and the Notch signaling pathway in angiogenesis [20,21] and suggest possible new targets for anti-angiogenic tumor therapy. 
Although clinical benefits of anti-VEGF therapy have been observed in patients with various types of cancers in terms of progression-free survival, the effects are variable, and acquired or innate resistance frequently occurs [22-25]. As an alternative, preclinical studies have demonstrated that blockade of DLL4 can have potent growth inhibitory effects on tumors that are resistant to anti-VEGF therapies [20, 26, 27]. Furthermore, simultaneous targeting of DLL4 and VEGF has shown additive anti-tumor effects compared to the effects of single agents in many tumor models [27]. Nevertheless, additional studies are warranted to ascertain the clinical validity of the combined treatment option.

Recent reports have demonstrated that DLL4 expression is related to the TNM stage, node stage, and distant metastasis, suggesting an association with poor prognosis in GC patients [28, 29]. By contrast, we did not find a correlation between DLL4 expression and clinicopathological parameters, including age, sex, smoking history, histological subtype, or tumor stage. This discrepancy between different studies may be related to limitations and differences in study designs. First, clinicopathological heterogeneity, such as distinct histological types, pathological stages, and different sample sizes among studies, may have affected the outcome of the analysis. Second, the difference in antibodies and IHC techniques used between studies may partly account for the different results. Third, the threshold of defining IHC-positive DLL4 expression differed among studies. Such unstandardized interpretation of the staining intensity and distribution, together with the use of various cut-off percentages to define DLL4 expression, ranging from $\geq 1 \%$ to $\geq 50 \%$, likely contributed to this apparent contradiction in correlations.

Although we found that high DLL4 expression was an independent prognostic factor of OS and DFS in GC patients at an advanced TNM stage (IIIb and IV), it is important to note that the small number of patients at stage III/IV in this cohort makes it difficult to provide a strong conclusion based on this result, owing to the low power of the analysis. Thus, large-scale studies are needed to confirm the role of DLL4 expression as a predictive biomarker for GC, especially for patients with advanced disease. Likewise, further analysis of the co-expression and clinical effects of various angiogenic factors such as CD45 and PECAM-1 would be valuable, as these factors may further promote tumor proliferation and angiogenesis within tumor vessels.

Our findings suggest that anti-DLL4 antibodies may be a novel therapy that could even be used even as an initial treatment for cancers of the gastrointestinal tract. ABL001 is a recently developed bispecific antibody targeting both VEGF and DLL4, representing a potent anti-cancer therapeutic antibody. This antibody links each C-terminal of an anti-VEGF antibody (bevacizumab-similar) with a DLL4-binding single-chain variable fragment ( $\mathrm{scFv}$ ) [12]. ABL001 was shown to more effectively inhibit human A549 lung cancer and SCH GC cells in xenograft models than a VEGF-targeting antibody [12]. Based on our previous observations on the preclinical anti-tumor efficacy of the DLL4 inhibitor and the present findings of the impact of DLL4 expression on the survival of GC patients, we are currently conducting a phase I trial with ABL001 (clinicaltrials.gov; NCT03292783).

In summary, high DLL4 expression was observed in $19.9 \%$ of GC patients and was significantly associated with poor survival following curative surgery. Given the substantial prevalence of the protein in the GC cohort with a poor prognosis, DLL4 may be a potential novel prognostic indicator of GC and offers a new strategy for the treatment of GC.

\section{Acknowledgments}

This work was supported by funding from the Basic Science Research Program through the National Research Foundation of Korea (NRF) funded by the Ministry of Education (2016R1A6A3A11932444) to K. Kim. This work was supported by funding from the Korean Health Technology R\&D Project, Ministry of Health \& Welfare, Republic of Korea (HI14C3418).

\section{Ethics Statement}

The ARTIST trial was approved by the institutional review board of the Samsung Medical Center (SMC, Seoul, Korea) and was conducted in accordance with the ethical principles of the Declaration of Helsinki and SMC guidelines. All protocols were approved by the ethics committees of SMC.

\section{Competing Interests}

The authors have declared that no competing interest exists.

\section{References}

1. Torre LA, Siegel RL, Ward EM, et al. Global Cancer Incidence and Mortality Rates and Trends--An Update. Cancer Epidemiol Biomarkers Prev. 2016; 25: $16-27$

2. Schmidt N, Peitz U, Lippert $\mathrm{H}$, et al. Missing gastric cancer in dyspepsia. Aliment Pharmacol Ther. 2005: 21: 813-820.

3. Wilke H, Muro K, Van Cutsem E, et al. Ramucirumab plus paclitaxel versus placebo plus paclitaxel in patients with previously treated advanced gastric or gastro-oesophageal junction adenocarcinoma (RAINBOW): a double-blind, randomised phase 3 trial. Lancet Oncol. 2014; 15: 1224-1235.

4. Hashizume H, Falcon BL, Kuroda $\mathrm{T}$, et al. Complementary actions of inhibitors of angiopoietin-2 and VEGF on tumor angiogenesis and growth. Cancer Res. 2010; 70: 2213-2223. 
5. You WK, Sennino B, Williamson CW, et al. VEGF and c-Met blockade amplify angiogenesis inhibition in pancreatic islet cancer. Cancer Res. 2011; 71: 4758-4768.

6. McKeage MJ, Kotasek D, Markman B, et al. Phase IB Trial of the Anti-Cancer Stem Cell DLL4-Binding Agent Demcizumab with Pemetrexed and Carboplatin as First-Line Treatment of Metastatic Non-Squamous NSCLC. Target Oncol. 2018; 13: 89-98.

7. Yen WC, Fischer MM, Axelrod F, et al. Targeting Notch signaling with a Notch2/Notch3 antagonist (tarextumab) inhibits tumor growth and decreases tumor-initiating cell frequency. Clin Cancer Res. 2015; 21: 2084-2095.

8. Ferrarotto R, Eckhardt G, Patnaik A, et al. A phase I dose-escalation and dose-expansion study of brontictuzumab in subjects with selected solid tumors. Ann Oncol. 2018; 29: 1561-1568.

9. Li JL, Sainson RC, Oon CE, et al. DLL4-Notch signaling mediates tumor resistance to anti-VEGF therapy in vivo. Cancer Res. 2011; 71: 6073-6083.

10. Hoey T, Yen WC, Axelrod F, et al. DLL4 blockade inhibits tumor growth and reduces tumor-initiating cell frequency. Cell Stem Cell. 2009; 5: 168-177.

11. Clarke JM, Hurwitz HI. Understanding and targeting resistance to anti-angiogenic therapies. J Gastrointest Oncol. 2013; 4: 253-263.

12. Lee D, Kim D, Choi YB, et al. Simultaneous blockade of VEGF and Dll4 by HD105, a bispecific antibody, inhibits tumor progression and angiogenesis. MAbs. 2016; 8: 892-904.

13. Park SH, Sohn TS, Lee J, et al. Phase III Trial to Compare Adjuvant Chemotherapy With Capecitabine and Cisplatin Versus Concurrent Chemoradiotherapy in Gastric Cancer: Final Report of the Adjuvant Chemoradiotherapy in Stomach Tumors Trial, Including Survival and Subset Analyses. J Clin Oncol. 2015; 33: 3130-3136.

14. $\mathrm{Hu} \mathrm{W}, \mathrm{Lu} \mathrm{C}$, Dong $\mathrm{HH}$, et al. Biological roles of the Delta family Notch ligand Dll4 in tumor and endothelial cells in ovarian cancer. Cancer Res. 2011; 71: 6030-6039.

15. Kontomanolis E, Panteliadou M, Giatromanolaki A, et al. Delta-like ligand 4 (DLL4) in the plasma and neoplastic tissues from breast cancer patients: correlation with metastasis. Med Oncol. 2014; 31: 945

16. Patel NS, Dobbie MS, Rochester M, et al. Up-regulation of endothelial delta-like 4 expression correlates with vessel maturation in bladder cancer. Clin Cancer Res. 2006; 12: 4836-4844

17. Jubb AM, Turley H, Moeller HC, et al. Expression of delta-like ligand 4 (Dll4) and markers of hypoxia in colon cancer. Br J Cancer. 2009; 101: 1749-1757.

18. Jubb AM, Browning L, Campo L, et al. Expression of vascular Notch ligands Delta-like 4 and Jagged-1 in glioblastoma. Histopathology. 2012; 60: 740-747.

19. Serafin V, Persano L, Moserle L, et al. Notch3 signalling promotes tumour growth in colorectal cancer. J Pathol. 2011; 224: 448-460.

20. Li JL, Sainson RC, Shi W, et al. Delta-like 4 Notch ligand regulates tumor angiogenesis, improves tumor vascular function, and promotes tumor growth in vivo. Cancer Res. 2007; 67: 11244-11253.

21. Yuan $\mathrm{X}, \mathrm{Wu} \mathrm{H}, \mathrm{Xu} \mathrm{H}$, et al. Notch signaling: an emerging therapeutic target for cancer treatment. Cancer Lett. 2015; 369: 20-27.

22. Ferrara N, Hillan KJ, Gerber HP et al. Discovery and development of bevacizumab, an anti-VEGF antibody for treating cancer. Nat Rev Drug Discov. 2004; 3: 391-400.

23. Bergers G, Hanahan D. Modes of resistance to anti-angiogenic therapy. Nat Rev Cancer. 2008; 8: 592-603.

24. Weis SM, Cheresh DA. Tumor angiogenesis: molecular pathways and therapeutic targets. Nat Med. 2011; 17: 1359-1370.

25. Casanovas $O$, Hicklin DJ, Bergers $G$, et al. Drug resistance by evasion of antiangiogenic targeting of VEGF signaling in late-stage pancreatic islet tumors. Cancer Cell. 2005; 8: 299-309.

26. Noguera-Troise I, Daly C, Papadopoulos NJ, et al. Blockade of Dll4 inhibits tumour growth by promoting non-productive angiogenesis. Nature. 2006; 444: 1032-1037.

27. Ridgway J, Zhang G, Wu Y, et al. Inhibition of Dll4 signalling inhibits tumour growth by deregulating angiogenesis. Nature. 2006; 444: 1083-1087.

28. Du X, Cheng Z, Wang YH, et al. Role of Notch signaling pathway in gastric cancer: a meta-analysis of the literature. World J Gastroenterol. 2014; 20: 9191-9199.

29. Ishigami S, Arigami T, Uenosono $\mathrm{Y}$, et al. Clinical implications of DLL4 expression in gastric cancer. J Exp Clin Cancer Res. 2013; 32: 46. 